\title{
Meta-analysis of epidemiological studies of association of two polymorphisms in the interleukin-10 gene promoter and colorectal cancer risk
}

\author{
Y.M. Zhang ${ }^{1}$, X.C. Zhou ${ }^{2}$, Z. Xu ${ }^{1}$ and C.J. Tang ${ }^{1}$ \\ ${ }^{1}$ Department of Oncology, Nanjing First Hospital, \\ Nanjing Medical University, Nanjing, Jiangsu, China \\ ${ }^{2}$ Department of Imaging and Nuclear Medicine, \\ Affiliated Hospital of Qinghai University, \\ Medical College of Qinghai University, XiNing, Qinghai, China \\ Corresponding author: C.J. Tang / Y.M. Zhang \\ E-mail: tangcuiju@tom.com / yangmeizhangnj@163.com
}

Genet. Mol. Res. 11 (3): 3389-3397 (2012)

Received October 13, 2011

Accepted March 16, 2012

Published September 25, 2012

DOI http://dx.doi.org/10.4238/2012.September.25.7

\begin{abstract}
In order to make a comprehensive assessment of the potential association between two genetic variants in the IL-10 gene promoter, -1082 A>G (rs1800896) and -592 C>A (rs1800872), and colorectal cancer (CRC) risk, we conduced a meta-analysis of seven epidemiological studies, which included 1469 colorectal cancer cases and 2566 controls. Neither of the two polymorphisms had any association with increased $\mathrm{CRC}$ risk in overall population [for rs1800896: odds ratio $(\mathrm{OR})=0.90,95 \%$ confidence interval $(95 \% \mathrm{CI})$ $=0.76-1.06$ in the dominant model and for rs1800872: OR $=1.06$, $95 \% \mathrm{CI}=0.91-1.23$ in the dominant model]. In subgroup analysis of the rs 1800896 polymorphism, the results did not change when the analyses were restricted to individual studies, or those fulfilling Hardy-Weinberg equilibrium, or according to the source of controls. For rs 1800872 ,
\end{abstract}


however, when stratifying by the source of controls, the A allele had a significant increased risk of CRC among studies with population-based controls in the codominant model $(\mathrm{AC} v s \mathrm{CC}$ : $\mathrm{OR}=1.30,95 \% \mathrm{CI}=$ 1.04-1.63) and dominant model (AA/AC vs CC: $\mathrm{OR}=1.25,95 \% \mathrm{CI}$ $=1.01-1.55)$. Based on this meta-analysis, we conclude that the IL-10 rs1800872 polymorphism could be a risk factor for CRC development among European populations. However, we found no association between the IL-10 rs1800896 polymorphism and CRC risk. Further studies, either with larger sample size or involving other SNPs and haplotypes of the IL-10 gene, are necessary to clarify the contribution of IL-10 genetic variations in colorectal carcinogenesis.

Key words: Interleukin-10; Colorectal cancer; Polymorphism; Meta-analysis

\section{INTRODUCTION}

Colorectal cancer (CRC) remains a major public health problem in the world, and is the third most common cancer in males and the second in females, with 26,470 deaths in males and 25,220 deaths in females expected to occur in 2012 in the United States (Siegel et al., 2012). It is widely accepted that cancer-associated inflammation promotes tumor growth and progression by inducing gene mutations, inhibiting apoptosis, and stimulating angiogenesis and cell proliferation (Baniyash, 2006; Vidal-Vanaclocha, 2009). Since the first case of inflammatory bowel disease associated with CRC was reported by Crohn and Rosenberg (1925), the association between chronic inflammation and CRC has been assessed by numerous laboratories worldwide (Lakatos and Lakatos, 2008; Xie and Itzkowitz, 2008; Triantafillidis et al., 2009). Accordingly, it is proposed that anti-inflammatory agents may protect colon and rectum from cancer development and metastasis. Convincing evidence from epidemiological studies has shown that aspirin and other non-steroidal inflammatory drugs may inhibit colorectal carcinogenesis (Tuynman et al., 2004; Chan et al., 2007).

Cytokines are important modulators for inflammatory response, whose functions can be crudely classified as pro-inflammatory [e.g., interleukin-1 (IL-1), tumor necrosis factor, interferon gamma (IFN- $\gamma$ ), IL-12, IL-18, and granulocyte-macrophage colony-stimulating factor] or anti-inflammatory [e.g., IL-4, IL-10, IL-13, IFN- $\alpha$, and transforming growth factor beta (Hanada and Yoshimura, 2002). In recent years, more and more investigators have devoted themselves to detecting single nucleotide polymorphisms (SNPs) and microsatellite polymorphisms within cytokine gene sequences, particularly within the promoter regions of these genes that have been found to be associated with cancer risks. Several of these polymorphisms may regulate levels of gene transcription and influence cancer susceptibility, development and prognosis. In this study, we focused on the role of IL-10 promoter gene polymorphisms in CRC predisposition.

The gene encoding IL-10 is located on chromosome 1 (1q31-1q32), comprising five exons (Eakdale et al., 1997). Two SNPs within the IL-10 gene promoter, rs1800896 (-1082 $\mathrm{A}>\mathrm{G})$ and rs $1800872(-592 \mathrm{C}>\mathrm{A})$, were reported to be associated with different IL-10 expression. The variant $\mathrm{G}$ allele at the -1082 locus was correlated with increased IL-10 expression 
and the variant A allele at the -592 locus was associated with decreased IL-10 expression in the in vitro experiment. -A1082G, -C819T, and -A592C combine to form three haplotypes; GCC, ACC, and ATA, which are linked to IL-10 expression level (Crawley et al., 1999; Crivello et al., 2006). Dysregulation of IL-10 gene expression and IL-10 promoter polymorphisms were reported to be associated with numerous autoimmune and infectious diseases and even malignancies (Howell and Rose-Zerilli, 2007).

Recently, several important polymorphic sites in the IL-10 gene, such as rs 1800896 and rs1800872, have been found to be involved in the pathogenesis of CRC. However, the results from different laboratories are conflicting. With the aim of providing a more precise estimation of the potential associations of the two polymorphisms with colorectal cancer risk, we performed a meta-analysis of the candidate eligible studies.

\section{MATERIAL AND METHODS}

\section{Identification of eligible studies}

PubMed, Embase, and Web of Science (updated to February 20, 2011) were searched by using the key words related to IL-10 gene polymorphisms and CRC (including colon, rectum or colorectal cancer; the full-search strategy is available from the authors on request). We also obtained the potentially relevant articles by hand-searching the reference lists for all original or review articles on this topic, as well as PubMed option 'Related Articles' in each research paper. The inclusion criteria were described as follows: i) published in English; ii) reports on human beings; iii) investigation of the association between promoter polymorphisms in IL-10 rs1800896, rs1800872 and cancer risk (including colon, rectum or colorectal cancers); iv) retrospective case-control studies or prospective cohort studies; v) containing available genotype frequency or odds ratio (OR) with its $95 \%$ confidence interval $(95 \% \mathrm{CI})$. Reports on patients known to have hereditary CRC syndromes, such as hereditary nonpolyposis CRC or familial adenomatous polyposis, as well as patients with precancerous lesions or adenomas were excluded (Gunter et al., 2006; Talseth et al., 2007; Lakatos and Lakatos, 2008). When there were multiple reports published for the same population, only the most recent or complete paper was selected in this meta-analysis.

\section{Search methods and data extraction}

Two investigators (Y.M. Zhang and $\mathrm{Z}$. Xu) independently searched the electronic databases (PubMed, Embase and Web of Science) and reviewed the abstracts or full texts to determine if they met the inclusion criteria. They also screened the references in the studies and PubMed option "Related Articles" to identify additional papers. Any discrepancy in studies' eligibility was adjudicated by Professor C.J. Tang. Data were extracted from all eligible publications independently by two of the authors (Y.M. Zhang and X.C. Zhou), where disagreements were resolved by discussion between the authors until a consensus was reached on every item. The following information was extracted from each study: first author name, publication year, country where this study was conducted and ethnicity, sum of genotype frequency for cases and controls, source of control groups, and genotyping methods. 


\section{Statistical analysis}

For our main analysis, we evaluated cancer risks with the heterozygote and rare homozygote, compared with the common homozygote [AG vs AA, GG vs AA in rs1800896 $(\mathrm{A}>\mathrm{G})$ and $\mathrm{AC} v s \mathrm{CC}, \mathrm{AA} v s \mathrm{CC}$ in $\mathrm{rs} 1800872(\mathrm{C}>\mathrm{A})]$, and then investigated the risks related to the dominant and recessive genotype model (AG/GG vs AA, GG vs AG/AA in rs1800896 and $\mathrm{AA} / \mathrm{AC}$ vs $\mathrm{CC}, \mathrm{AA}$ vs $\mathrm{AC} / \mathrm{CC}$ in rs 1800872), assuming the dominant and recessive effects of the variant $\mathrm{G}$ and $\mathrm{A}$ alleles between cases and controls, respectively. For each study, HardyWeinberg equilibrium (HWE) was also evaluated using the goodness-of-fit chi-square test. Stratified analysis was further performed to evaluate the association between IL-10 gene polymorphisms and CRC risk. The strength of the associations between the IL-10 gene promoter polymorphisms and cancer risk was presented as the OR and corresponding $95 \% \mathrm{CI}$. The value of pooled OR was tested using the Z-test and was considered to be statistically significant at $\mathrm{P}<0.05$ (Breslow and Day, 1987). The pooled OR was calculated by a fixed-effects model (using the Mantel-Haenszel method) or a random-effect model (using the DerSimonian and Laird method) according to the heterogeneity among studies (Midgette et al., 1994). Sensitivity analysis was conducted by omitting each study to assess the stability of the results. Funnel plots and the Egger linear regression test were used to provide diagnosis of the potential publication bias. All statistical analyses were conducted using Stata/SE version 10.0 (Stata Corporation, College Station, TX, USA).

\section{RESULTS}

\section{Characteristics of eligible studies}

A total of eight studies meeting the inclusion criteria were retrieved from the searched databases. In the eight papers, three polymorphisms in the IL-10 gene promoter were analyzed (at positions -1082, -819 and -592) and only three studies investigated the association of -819 SNPs with CRC. As the allele or genotype frequencies of the IL-10 gene -819 polymorphism were not reported in the study by Lee et al. (2010), we excluded it from the analysis. Finally, the meta-analysis was performed based on seven studies with a total of 1469 CRC cases and 2566 controls from different countries in Europe, for assessing the associations of two polymorphisms in the IL-10 gene (rs1800896, rs 1800872) with CRC risks (Macarthur et al., 2005; Crivello et al., 2006; Cozar et al., 2007; Vogel et al., 2007; Cacev et al., 2008; Wilkening et al., 2008; Tsilidis et al., 2009). The study selection process is shown in Figure 1. In the seven articles, three of them were cohort studies and the controls were selected from the same cohort as the corresponding case, without any previous cancer diagnosis (except for carcinoma in situ of cervix uteri or non-melanoma skin cancer). The four other case-control studies recruited healthy individuals as controls. In all papers, the distribution of the genotypes in the control group was consistent with HWE except one study (Wilkening et al., 2008) (Table 1).

\section{Meta-analysis}

The main results of the meta-analysis of the association between two polymorphisms and CRC risk are shown in Table 2. Neither of the two polymorphisms had any association 
with increased CRC risk in the overall population. In subgroup analysis of rs 1800896 polymorphism, the results did not change when the analyses were restricted to the studies fulfilling HWE, or according to the source of controls. For rs1800872, however, when restricted to studies with healthy population-based controls, variant $\mathrm{A}$ allele carriers had a significant increased risk of $\mathrm{CRC}$ in the codominant model $(\mathrm{AC} v \mathrm{CC}$ : $\mathrm{OR}=1.30,95 \% \mathrm{CI}=1.04-1.63)$ and dominant model (AA/AC vs $\mathrm{CC}$ : $\mathrm{OR}=1.25,95 \% \mathrm{CI}=1.01-1.55)$, although a similar association was not observed in other models (Table 2).

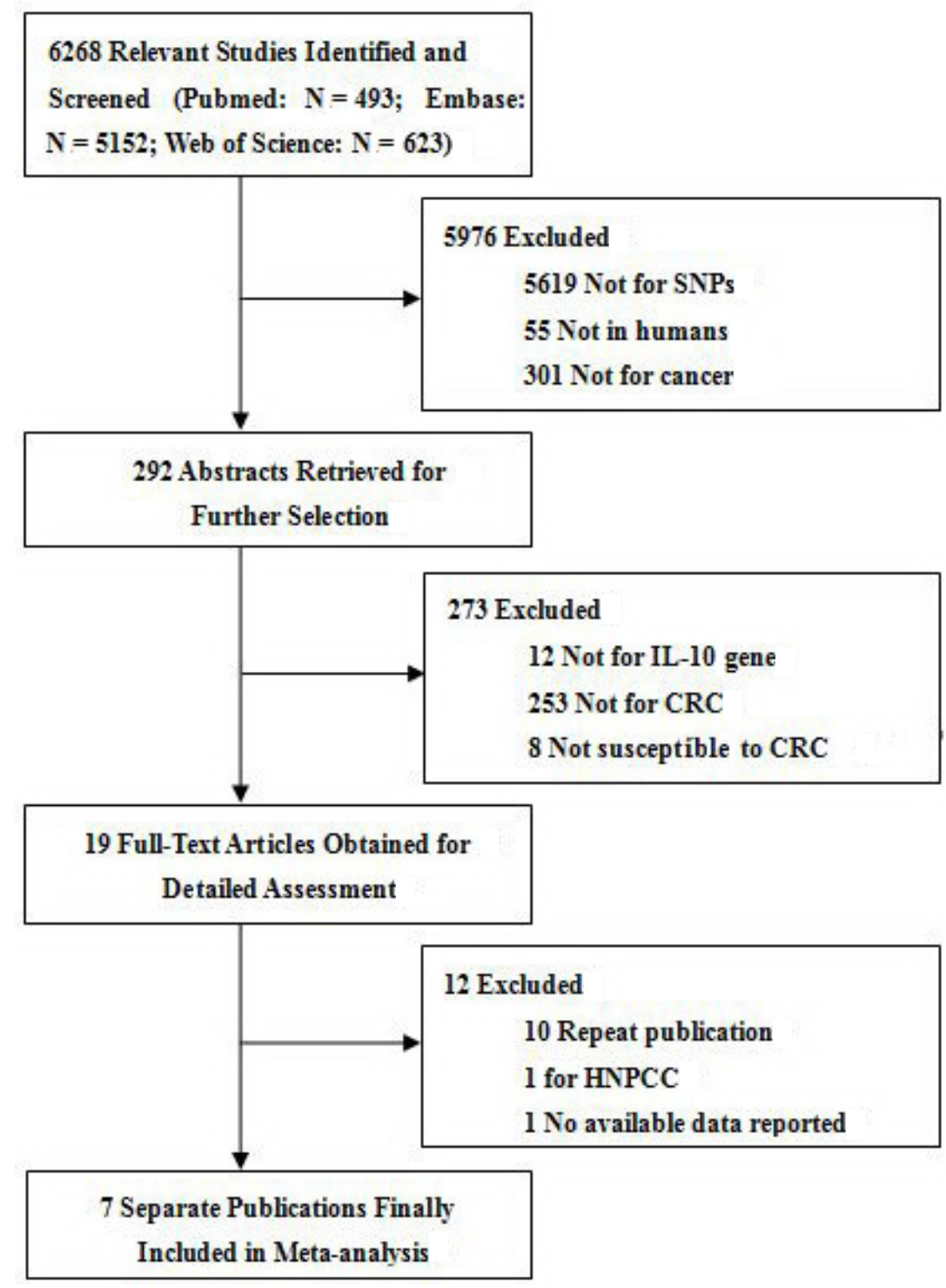

Figure 1. Flow diagram of included/excluded studies. SNPS = single nucleatide polymorphisms; CRC = colorectal cancer; HNPCC $=$ hereditary nonpolyposis colorectal cancer. 
Table 1. Characterics of studies included in the meta-analysis.

\begin{tabular}{|c|c|c|c|c|c|c|c|c|c|c|c|c|}
\hline \multirow[t]{2}{*}{ SNP } & \multirow[t]{2}{*}{ Study (year) } & \multirow[t]{2}{*}{ Country (ethnicity) } & \multirow{2}{*}{$\begin{array}{c}\text { Source of } \\
\text { controls }\end{array}$} & \multirow{2}{*}{$\begin{array}{l}\text { Genotyping } \\
\text { method }\end{array}$} & \multicolumn{3}{|c|}{ Cases } & \multicolumn{3}{|c|}{ Controls } & \multirow[t]{2}{*}{ HWE } & \multirow[t]{2}{*}{ MAF } \\
\hline & & & & & AA & AG & GG & $\mathrm{AA}$ & $\mathrm{AG}$ & GG & & \\
\hline \multirow[t]{7}{*}{ rs1800896 } & Tsilidis (2009) & USA (European) & $\mathrm{CB}$ & TaqMan & 69 & 101 & 35 & 98 & 187 & 87 & 0.903 & 0.485 \\
\hline & Wilkening (2008) & Sweden (European) & $\mathrm{CB}$ & TaqMan & 83 & 146 & 75 & 164 & 283 & 132 & 0.639 & 0.472 \\
\hline & Cacev (2008) & Croatia (European) & PB & PCR-RFLP & 54 & 86 & 20 & 43 & 92 & 25 & 0.038 & 0.444 \\
\hline & Cozar (2007) & Spain (European) & PB & TaqMan & 42 & 62 & 22 & 58 & 87 & 30 & 0.787 & 0.420 \\
\hline & Crivello (2006) & Italy (European) & PB & ARMS-PCR & 16 & 34 & 12 & 38 & 60 & 26 & 0.797 & 0.452 \\
\hline & Macarthur (2005) & Scotland (European) & PB & TaqMan & 61 & 125 & 71 & 86 & 202 & 115 & 0.877 & 0.536 \\
\hline & & & & & $\mathrm{CC}$ & $\mathrm{AC}$ & $\mathrm{AA}$ & $\mathrm{CC}$ & $\mathrm{AC}$ & $\mathrm{AA}$ & & \\
\hline \multirow[t]{6}{*}{ rs1800872 } & Tsilidis (2009) & USA (European) & $\mathrm{CB}$ & TaqMan & 123 & 71 & 9 & 213 & 131 & 17 & 0.580 & 0.229 \\
\hline & Cacev (2008) & Croatia (European) & PB & PCR-RFLP & 83 & 64 & 13 & 104 & 52 & 4 & 0.399 & 0.188 \\
\hline & Vogel (2007) & Danish (European) & $\mathrm{CB}$ & TaqMan & 224 & 109 & 22 & 455 & 256 & 42 & 0.450 & 0.226 \\
\hline & Cozar (2007) & Spain (European) & PB & TaqMan & 52 & 41 & 2 & 98 & 63 & 14 & 0.394 & 0.260 \\
\hline & Crivello (2006) & Italy (European) & PB & ARMS-PCR & 31 & 28 & 3 & 69 & 48 & 7 & 0.719 & 0.250 \\
\hline & Macarthur (2005) & Scotland (European) & PB & TaqMan & 151 & 99 & 8 & 248 & 133 & 22 & 0.456 & 0.220 \\
\hline
\end{tabular}

$\overline{\mathrm{SNP}}=$ single nucleotide polymorphism; $\mathrm{PB}=$ population-based study; $\mathrm{CB}=$ cohort-based study; ARMS $=$ amplification refractory mutation system; $\mathrm{PCR}=$ polymerase chain reaction; RFLP $=$ restriction fragment length polymorphism; HWE = Hardy-Weinberg equilibrium; MAF = minor allele frequency.

\begin{tabular}{|c|c|c|c|c|c|c|}
\hline \multirow[t]{2}{*}{ Analysis model } & \multicolumn{3}{|c|}{ rs 1800896 OR $(95 \% \mathrm{CI})$} & \multicolumn{3}{|c|}{ rs 1800872 OR $(95 \% \mathrm{CI})$} \\
\hline & $\begin{array}{l}\text { Overall } \\
(\mathrm{N}=6 ; \\
1114 / 1813)^{\mathrm{a}}\end{array}$ & $\begin{array}{l}\text { Population-based } \\
(\mathrm{N}=4 \\
480 / 666)^{\mathrm{a}}\end{array}$ & $\begin{array}{l}\text { Fulfilling HWE } \\
(\mathrm{N}=5 \\
974 / 1678)^{\mathrm{a}}\end{array}$ & $\begin{array}{l}\text { Overall } \\
(\mathrm{N}=6 ; \\
1133 / 1976)^{\mathrm{a}}\end{array}$ & $\begin{array}{l}\text { Population-based } \\
(\mathrm{N}=4 ; \\
911 / 1692)^{\mathrm{a}}\end{array}$ & $\begin{array}{l}\text { Fulfilling HWE } \\
(\mathrm{N}=6 ; \\
1133 / 1976)^{\mathrm{a}}\end{array}$ \\
\hline $\begin{array}{l}\text { Codominant (het. } \\
v s \text { common hom.) }\end{array}$ & $\begin{array}{l}0.91 \\
(0.76-1.08)\end{array}$ & $\begin{array}{l}0.91 \\
(0.71-1.17)\end{array}$ & $\begin{array}{l}0.93 \\
(0.77-1.13)\end{array}$ & $\begin{array}{l}1.07 \\
(0.92-1.25)\end{array}$ & $\begin{array}{l}1.30 \\
(1.04-1.63)\end{array}$ & $\begin{array}{l}1.07 \\
(0.92-1.25)\end{array}$ \\
\hline $\mathrm{P} / \mathrm{P}_{\mathrm{h}}$ & $0.283 / 0.688$ & $0.452 / 0.594$ & $0.483 / 0.666$ & $0.381 / 0.268$ & $0.021 / 0.874$ & $0.381 / 0.268$ \\
\hline Codominant (rare hom. & 0.87 & 0.87 & 0.90 & 0.97 & 0.92 & 0.97 \\
\hline vs common hom.) & $(0.70-1.08)$ & $(0.64-1.18)$ & $(0.71-1.13)$ & $(0.69-1.36)$ & $(0.31-2.73)^{\mathrm{c}}$ & $(0.69-1.36)$ \\
\hline $\mathrm{P} / \mathrm{P}_{\mathrm{h}}$ & $0.202 / 0.350$ & $0.376 / 0.757$ & $0.347 / 0.313$ & $0.852 / 0.073$ & $0.882 / 0.019$ & $0.852 / 0.073$ \\
\hline Dominant (rare hom. & 0.90 & 0.90 & 0.92 & 1.06 & 1.25 & 1.06 \\
\hline + het. vs common hom.) & $(0.76-1.06)$ & $(0.71-1.14)$ & $(0.77-1.10)$ & $(0.91-1.23)$ & $(1.01-1.55)$ & $(0.91-1.23)$ \\
\hline $\mathrm{P} / \mathrm{P}_{\mathrm{h}}$ & $0.198 / 0.465$ & $0.372 / 0.579$ & $0.383 / 0.444$ & $0.452 / 0.213$ & $0.044 / 0.428$ & $0.452 / 0.213$ \\
\hline Recessive (rare hom. & 0.93 & 0.92 & 0.96 & 0.94 & 0.83 & 0.94 \\
\hline$v s$ het. + common hom.) & $(0.79-1.10)$ & $(0.73-1.16)$ & $(0.80-1.14)$ & $(0.68-1.32)$ & $(0.29-2.35)^{\mathrm{c}}$ & $(0.68-1.32)$ \\
\hline $\mathrm{P} / \mathrm{P}_{\mathrm{h}}$ & $0.394 / 0.867$ & $0.494 / 0.725$ & $0.626 / 0.891$ & $0.730 / 0.075$ & $0.427 / 0.025$ & $0.730 / 0.075$ \\
\hline
\end{tabular}

het. = heterozygous; hom. $=$ homozygous; $95 \% \mathrm{CI}=95 \%$ confidence interval; HWE $=$ Hardy-Weinberg equilibrium. ${ }^{a}$ Study number, number of cases and controls. $\mathrm{P}_{\mathrm{h}}$ denotes $\mathrm{P}$ value of the Q-test for heterogeneity test.

In addition, we explored the publication bias by applying Begg's funnel plot and the Egger test. Funnel plots did not demonstrate an evident asymmetry, and the P value of the Egger test indicated the absence of publication bias in all genetic comparisons (data not shown). Moreover, the nonparametric trim and fill method was performed to evaluate the influence of any single study on the pooled OR of rs 1800896 and rs 1800872 , respectively. The results showed that there was no individual study qualitatively affecting the overall OR, since there was no difference by omitting any individual report, which suggested that the findings of this meta-analysis were relatively stable. 


\section{DISCUSSION}

To date, lines of research have investigated the contributions of IL-10 gene polymorphisms to the predisposition to different cancer types, such as oral, stomach, liver, breast, ovarian, cervical, prostate, and so on (Howell and Rose-Zerilli, 2007). In our study, we performed the first meta-analysis to explore the role of genetic polymorphisms of the IL-10 promoter gene in CRC susceptibility. The results showed that the IL-10 rs1800872 polymorphism may be a risk factor for CRC development among European populations, although there is no association between the IL-10 rs1800896 polymorphism and CRC risk.

Local production of cytokines within the tumor microenvironment is an important modulator during tumorigenesis (De Vita et al., 1999). A zoological study conducted by Sturlan et al. (2001) indicated that IL-10-deficient mice can develop chronic enterocolitis and CRC. Moreover, a statistically significant decrease in IL-10 mRNA expression in tumor tissue of patients with colon cancer compared to normal mucous tissue was observed (Cacev et al., 2008). Meanwhile, there is evidence showing that the two candidate IL-10 SNPs (rs1800872 and rs1800896) located in the promoter region are consistently associated with changes in IL-10 transcriptional activity and expression (Crawley et al., 1999; Crivello et al., 2006). Therefore, it is assumed that IL-10 gene polymorphisms may affect susceptibility to CRC by influencing IL-10 expression. This hypothesis was not well confirmed by our finding of a lack of association between the rs 1800896 polymorphism and CRC risk. However, our finding of the effect of the rs 1800872 polymorphism on CRC carcinogenesis could provide appropriate supports for the hypothesis. The potential explanation for our results may be attributed to the dual functions of IL-10 (anti-inflammation and anti-angiopoiesis), and the complex regulatory network modulating IL-10 gene expression. Some researchers have suggested that about $75 \%$ of inter-individual variations in IL-10 gene expression may be due to the genetic variations (Eskdale et al., 1998), where the promoter polymorphisms may play an important role. In addition to the two candidate SNPs in this meta-analysis, other SNPs at loci -819, -1349, $-3575,-459,+19$, and +117 , and two microsatellite polymorphisms have been identified in the IL-10 gene promoter and reported to have some impacts on the susceptibility to malignancies and antoimmune disease (Zheng et al., 2001; Bulpitt et al., 2004). It has also been found that the $-1082 \mathrm{~A},-819 \mathrm{C},-592 \mathrm{C}$, and $+117 \mathrm{~T}$ haplotypes accelerate the progression of chronic infection induced by hepatitis B virus, especially to hepatocellular carcinoma development through the upregulation of IL-10 gene expression (Shin et al., 2003). The above findings prompt investigators to perform more comprehensive haplotype-based or multiple SNP-based studies rather than single polymorphism-based observations, which may offer more sufficient information on contributions of IL-10 genetic variation to CRC susceptibility, development and prognosis. Unfortunately, a haplotype-based meta-analysis cannot be conducted here according to the current data extracted from the eligible publications.

It is important to note that some limitations existed in the present meta-analysis. First, the combined ORs were based on individual unadjusted estimates. Second, there are two studies including less than 100 cases in our meta-analysis (Crawley et al., 1999; Crivello et al., 2006), which may cause unreliable conclusions, due to type 1 or type 2 errors. Third, the effects of the $-1082 \mathrm{G}$ and $-592 \mathrm{C}$ alleles may be masked by the potential interactions, namely gene-gene, gene-environment and even different polymorphic loci of the same gene, making it difficult to exclude the possibility of potential risk contribution of these two polymorphisms (rs1800896 and 
rs1800872) in the IL-10 gene to CRC risk. In addition, it is possible that the polymorphisms of interest may be associated with risk factors for CRC, including body mass index, physical activity, social class, alcohol intake, total energy intake, and smoking status, which were regarded as potential confounders of the association between each of the polymorphisms and CRC. Therefore, either positive or negative results on the relationship between SNPs under investigation with CRC obtained from different reviewed studies should be treated with caution.

Several potential factors that may lead to bias should also be acknowledged in this field. First, publication bias may have been active, even though the use of a statistical test did not show it here. Second, only one study specifically mentioned that genotyping was blinded to case/control status (Tsilidis et al., 2009), one study performed some form of genotyping quality control (Cacev et al., 2008), and different genotyping methods were conducted in the relevant studies. This discrepancy between reports highlights the need for implementing a more rigorous study design in future research. Finally, meta-analysis remains a retrospective research that is subject to the methodological deficiencies of the studies included. The use of explicit methods for study selection, data extraction and data analysis may help us minimize the likelihood of this bias.

In any event, the current meta-analysis suggests that the rs 1800872 polymorphism may be a risk factor for CRC, while the rs 1800896 polymorphism has no effect on the susceptibility to CRC. Further studies, either with large sample size or with respect to other SNPs and haplotypes in the IL-10 gene, are necessary to clarify the contribution of IL-10 genetic variations in the carcinogenesis, development and prognosis of CRC.

\section{REFERENCES}

Baniyash M (2006). Chronic inflammation, immunosuppression and cancer: new insights and outlook. Semin. Cancer Biol. 16: 80-88.

Breslow NE and Day NE (1987). Statistical methods in cancer research. Volume II - The design and analysis of cohort studies. IARC Sci. Publ.1-406.

Bulpitt EA, Baynesc C, Dunning AM, Evans PR, et al. (2004). Investigation of interleukin-10 and vascular endothelial growth factor single nucleotide polymorphisms in predisposition to breast cancer. Genes Immun. 5: S1-S53.

Cacev T, Radosevic S, Krizanac S and Kapitanovic S (2008). Influence of interleukin-8 and interleukin-10 on sporadic colon cancer development and progression. Carcinogenesis 29: 1572-1580.

Chan AT, Ogino S and Fuchs CS (2007). Aspirin and the risk of colorectal cancer in relation to the expression of COX-2. N. Engl. J. Med. 356: 2131-2142.

Cozar JM, Romero JM, Aptsiauri N, Vazquez F, et al. (2007). High incidence of CTLA-4 AA (CT60) polymorphism in renal cell cancer. Hum. Immunol. 68: 698-704.

Crawley E, Kay R, Sillibourne J, Patel P, et al. (1999). Polymorphic haplotypes of the interleukin-10 5' flanking region determine variable interleukin-10 transcription and are associated with particular phenotypes of juvenile rheumatoid arthritis. Arthritis Rheum. 42: 1101-1108.

Crivello A, Giacalone A, Vaglica M, Scola L, et al. (2006). Regulatory cytokine gene polymorphisms and risk of colorectal carcinoma. Ann. N. Y. Acad. Sci. 1089: 98-103.

Crohn BB and Rosenberg H (1925). The sigmoidoscopic picture of chronic ulcerative colitis (non-specific). Am. J. Med. Sci. 170: 220-228.

De Vita F, Orditura M, Galizia G, Romano C, et al. (1999). Serum interleukin-10 levels in patients with advanced gastrointestinal malignancies. Cancer 86: 1936-1943.

Eskdale J, Kube D, Tesch H and Gallagher G (1997). Mapping of the human IL10 gene and further characterization of the 5' flanking sequence. Immunogenetics 46: 120-128.

Eskdale J, Gallagher G, Verweij CL, Keijsers V, et al. (1998). Interleukin 10 secretion in relation to human IL-10 locus haplotypes. Proc. Natl. Acad. Sci. U. S. A. 95: 9465-9470.

Gunter MJ, Canzian F, Landi S, Chanock SJ, et al. (2006). Inflammation-related gene polymorphisms and colorectal adenoma. Cancer Epidemiol. Biomarkers Prev. 15: 1126-1131. 
Hanada T and Yoshimura A (2002). Regulation of cytokine signaling and inflammation. Cytokine Growth Factor Rev. 13: 413-421.

Howell WM and Rose-Zerilli MJ (2007). Cytokine gene polymorphisms, cancer susceptibility, and prognosis. J. Nutr. 137: 194S-199S.

Lakatos PL and Lakatos L (2008). Risk for colorectal cancer in ulcerative colitis: changes, causes and management strategies. World J. Gastroenterol. 14: 3937-3947.

Lee YS, Choi HB, Lee IK, Kim TG, et al. (2010). Association between interleukin-4R and TGF-beta1 gene polymorphisms and the risk of colorectal cancer in a Korean population. Colorectal Dis. 12: 1208-1212.

Macarthur M, Sharp L, Hold GL, Little J, et al. (2005). The role of cytokine gene polymorphisms in colorectal cancer and their interaction with aspirin use in the northeast of Scotland. Cancer Epidemiol. Biomarkers Prev. 14: 1613-1618.

Midgette AS, Wong JB, Beshansky JR, Porath A, et al. (1994). Cost - Effectiueness of streptokinase for acute myocardial infarction: A combined meta-analysis and decision analysis of the effects of infarct location and of likelihood of infarction. Med. Decis. Making 14: 108-117.

Shin HD, Park BL, Kim LH, Jung JH, et al. (2003). Interleukin 10 haplotype associated with increased risk of hepatocellular carcinoma. Hum. Mol. Genet. 12: 901-906.

Siegel R, Naishadham D and Jemal A (2012). Cancer statistics, 2012. CA Cancer J. Clin. 62: 10-29.

Sturlan S, Oberhuber G, Beinhauer BG, Tichy B, et al. (2001). Interleukin-10-deficient mice and inflammatory bowel disease associated cancer development. Carcinogenesis 22: 665-671.

Talseth BA, Meldrum C, Suchy J, Kurzawski G, et al. (2007). Lack of association between genetic polymorphisms in cytokine genes and disease expression in patients with hereditary non-polyposis colorectal cancer. Scand. $J$. Gastroenterol. 42: 628-632.

Triantafillidis JK, Nasioulas G and Kosmidis PA (2009). Colorectal cancer and inflammatory bowel disease: epidemiology, risk factors, mechanisms of carcinogenesis and prevention strategies. Anticancer Res. 29: 2727-2737.

Tsilidis KK, Helzlsouer KJ, Smith MW, Grinberg V, et al. (2009). Association of common polymorphisms in IL10, and in other genes related to inflammatory response and obesity with colorectal cancer. Cancer Causes Control. 20: 1739-1751.

Tuynman JB, Peppelenbosch MP and Richel DJ (2004). COX-2 inhibition as a tool to treat and prevent colorectal cancer. Crit. Rev. Oncol. Hematol. 52: 81-101.

Vidal-Vanaclocha F (2009). Inflammation in the molecular pathogenesis of cancer and atherosclerosis. Reumatol. Clin. 5S1: 40-43.

Vogel U, Christensen J, Dybdahl M, Friis S, et al. (2007). Prospective study of interaction between alcohol, NSAID use and polymorphisms in genes involved in the inflammatory response in relation to risk of colorectal cancer. Mutat. Res. 624: 88-100.

Wilkening S, Tavelin B, Canzian F, Enquist K, et al. (2008). Interleukin promoter polymorphisms and prognosis in colorectal cancer. Carcinogenesis 29: 1202-1206.

Xie J and Itzkowitz SH (2008). Cancer in inflammatory bowel disease. World J. Gastroenterol. 14: 378-389.

Zheng C, Huang D, Liu L, Wu R, et al. (2001). Interleukin-10 gene promoter polymorphisms in multiple myeloma. Int. J. Cancer 95: 184-188. 